\title{
Experiment planning using the high level parameter concept
}

\author{
Heike Riemann ${ }^{\mathrm{a},{ }^{*}}$, Torsten Bluhm ${ }^{\mathrm{a}}$, Peter Heimann ${ }^{\mathrm{b}}$, Christine Hennig $^{\mathrm{a}}$, \\ Georg Kühner ${ }^{a}$, Hugo Kroiss ${ }^{b}$, Heike Laqua ${ }^{a}$, Marc Lewerentz ${ }^{a}$, Josef Maier ${ }^{b}$, \\ Jörg Schacht ${ }^{\mathrm{a}}$, Anett Spring ${ }^{\mathrm{a}}$, Andreas Werner ${ }^{\mathrm{a}}$, Manfred Zilker ${ }^{\mathrm{b}}$ \\ ${ }^{a}$ Max-Planck-Institut für Plasmaphysik, EURATOM Association, Teilinstitut Greifswald, \\ Wendelsteinstraße 1, D-17491 Greifswald, Germany \\ ${ }^{b}$ Max-Planck-Institut für Plasmaphysik, EURATOM Association, Boltzmannstraße 2, \\ D-85748 Garching, Germany \\ "Corresponding author; Tel.: +49-3834-88-2225; Fax: +49-3834-88-2509; \\ E-mail address: Heike.Riemann@ipp.mpg.de
}

\begin{abstract}
One discharge at the steady-state experiment Wendelstein 7-X will last up to 30 min and will be divided into many elementary experimental program phases, so-called segments. Physics programs need to be planned and technical parameters need to be adjusted well before the discharge. The technical parameters provide a complete description for the behavior of all participating components.
\end{abstract}

Physicists, however, aim at specifying the state and behavior of both plasma and machine in terms of physics. Therefore, an abstraction layer above the technical parameters has been developed, the so-called high level parameters. These specify a subset of the technical parameters on a higher, more physics-oriented level and are therefore used to encapsulate technical details. During ordinary program planning, the physicists and engineers only see and work with these high level (physics) parameters.

In comparison to shot-based and other existing experiments, this is an entirely new scientifically oriented method to plan experiments in terms of physics parameters (supported by tools) which are automatically mapped onto technical parameters. Besides the introduction of the high level parameter concept, this paper shows the planning of experiment programs with high level parameters for the example of the WEGA (a small stellarator experiment) magnetic field. For this case, details about defining and editing these parameters as well as about the mapping of high onto low level (technical) parameters will be given. Furthermore, the support of these processes by specialized tools is described. In addition, other fields of high level parameter applications will be mentioned.

Keywords: High level parameter; Physics parameter; Abstraction layer; Transformation; Planning experiments; Scientifically oriented 


\section{Introduction}

Wendelstein 7-X will be a steady-state experiment with a discharge duration up to $30 \mathrm{~min}$. In order to investigate different physics objectives, one discharge (or experiment program) is separated into scenarios. Each scenario is fragmented into arbitrary time intervals, so-called segments. The desired state and behavior of all W7-X components during the segment execution is given in a segment description. Segment descriptions are basically lists of parameter sets for the system components of one segment.

The W7-X system components are hierarchically structured: one project (experiment level or laboratory) contains components, which in turn contain subcomponents [1]. The system components need their full technical (low level) parameter set for each segment to operate. Because almost no manual interaction with the hardware is possible during the discharge, all parameters have to be set up for each segment before the discharge and will be switched to automatically. All parameter descriptions as well as other information about system configuration and experiment program specification are stored in the W7-X configuration database [1].

Although experiment planning and control in the past has often been performed with scientific meaningful quantities, these plans were rarely available in a format that could directly be used to execute the experiment program. The transformation from the scientific meaningful quantities to the technical parameters, which are needed to run an experiment, was established either in head, on the basis of tables, by modeling experience or by particular transformation functions being exposed as standalone applications. Wendelstein 7-X employs the more generic high level parameter concept for a straight forward experiment planning.

\section{High level parameter concept}

The purposes of the high level parameter (HLP) concept are to enable physicists to specify the behavior and state of the plasma and machine in terms of physics values (supported by tools), to reuse patterns and to make the sum of experiences persistent. High level parameters specify an abstraction of technical parameters on a physics level and can therefore be used to encapsulate technical details. Moreover, they can be used as filter function for relevant low level parameters as well as for parameters with marginal requirement for abstraction, such as for tests of simple systems or subsystems, see Fig.1 . In both cases unnecessary details are filtered-out, leading to a reduction of complexity.

During ordinary program planning, the diagnosticians and technicians only see and work with these high level parameters. For experiment execution they have to be transformed into technical parameters. Depending on the level of abstraction, the transformation procedures range from simple one-to-one to very complicated functions [2]. 


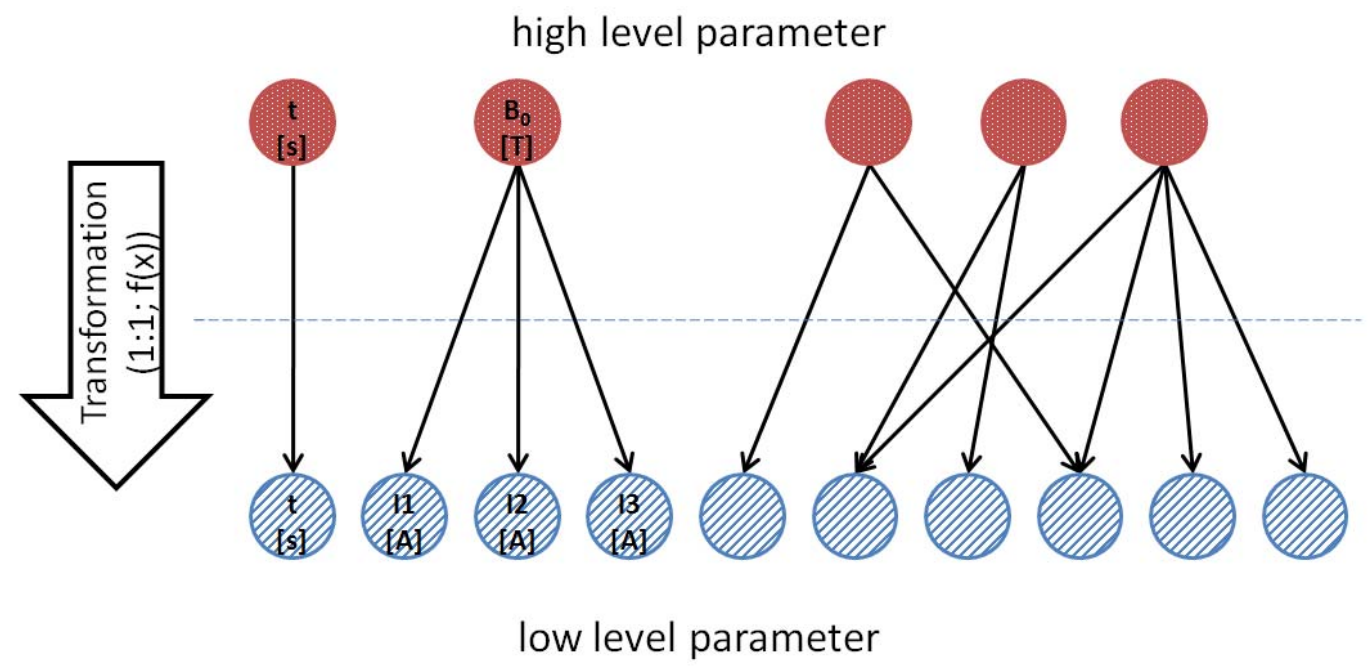

Fig. 1. Relations of high and low level parameters.

High level parameters may have mutual dependencies according to the abstraction level as well as to the chosen abstraction. As a consequence, a change of one parameter value may influence the validity range of other parameters, which has to be expressed by appropriate functions for their upper and lower boundaries.

Since many high level parameters exist for each segment description, they are structured in order to reduce complexity. The structure determines the view on the high level parameters and is used to build a graphical user interface for editing them. Structuring is done by a hierarchy of sequences (collection of high level parameters), choices (alternatives for defining high level parameters) and parameters. Details can be found in ref. [2].

In order to meet the requirement for flexibility in an experimental environment, content and description of high level parameters are separated. The contents are the concrete parameter values. The description contains specification and presentation information, e.g. name, identification and properties like minimum, maximum and default values, unit and tooltip. The structure is exclusively determined by the descriptions, the parameter set contents have a flat representation. This separation makes restructuring possible without affecting parameters already used, which is necessary e.g. when the view onto the high level parameters has changed.

However, system components need their complete low level parameter set to operate correctly. This is guaranteed by a user-defined transformation function (Section 3.3) [2] mapping high onto low level parameters. Since not all low level parameters are specified by the high level parameters, a pattern is associated with each high level segment description. This pattern contains a complete set of default low level parameters for the system components of one segment. A copy of this pattern will be generated, partially overwritten with values mapped from the high level parameters and an executable segment description emerges (see Fig. 2). 


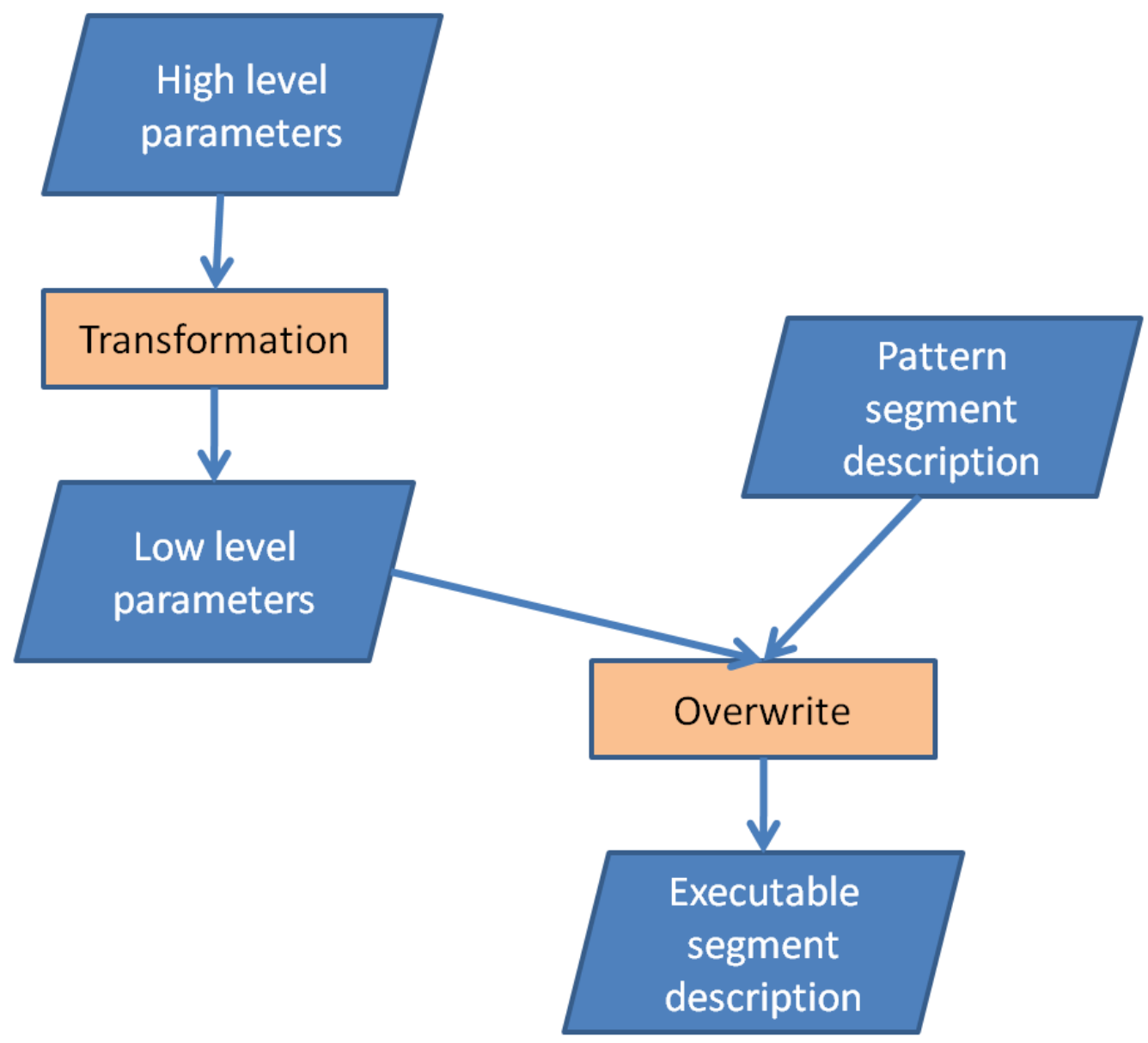

Fig. 2. From high level parameters to an executable segment description.

A configuration change of W7-X components can lead to many changes in low and high level parameters of all segment descriptions. This requires a migration strategy for the existing segment descriptions in the configuration database. A wizard guiding through the process step by step is under development. Using high level parameters, mainly pattern segment descriptions have to be adapted in connection with a configuration change. Where necessary, e.g. in case of additional or invalid high level parameters, the high level parameters themselves have to be adapted. In order to reuse existing high level parameter descriptions, the invalid high level parameters will be marked as obsolete instead of deleting them. Having finished modifying the pattern segment descriptions and high level parameters, the transformation and overwrite process create a modified executable segment description.

\section{Planning experiment programs with high level parameters}

The planning of experiment programs with high level parameters will be shown using the example of WEGA magnetic field. WEGA is a small stellarator experiment in Greifswald and is used as a test bed for diagnostics prototypes as well as for the W7-X control and data acquisition (CoDaC) system - and therefore also to prove and refine the high level parameter concept. An implementation for magnetic configuration control with high level parameters is now in use at WEGA. To enable work with high level parameters with the support of specialized tools, these parameters have to be identified by the diagnosticians. According to ref. [3] the magnetic field strength on axis $B_{0}$, the major plasma radius $R$ and the rotational transform iota (corresponding to the safety factor $q$ in tokamaks) have been chosen as high 
level parameter set for magnetic field control. These quantities are essentially plasma physics control parameters, which can be uniquely transformed into technical parameters, the coil currents for toroidal, helical and vertical field coils by employing fast function parametrisation methods as described in ref. [3]. Beside the physical quantities mentioned above, even magnetic island positions could be used as control parameters, which, however, would replace the rotational transform. In this way, alternative input parameter sets can be defined for the magnet power supplies and allow the selection of the suitable set according to the envisaged physics scenario.

\subsection{Setting up high level parameters (component level)}

Once high level parameters have been identified for one component, their descriptions have to be set up in order to edit the content afterwards. Setting up high level parameter descriptions includes creating their structure and specifying the transformation functions as well as the corresponding target paths to low level parameters. This infrequent task is supported by an expert tool, the configuration editor (see screenshot in Fig.3).

For example, to set up the magnetic configuration for WEGA as high level parameters the following steps have to be done:

1) Create a choice with different possibilities to specify the magnetic configuration, e.g. “ramp to magnetic configuration”, "keep setpoint values” or “de-energize all coils” (see Fig. 3).

2) Create high level parameter descriptions for each choice entry. For example, for "ramp to magnetic configuration" the following high level parameter descriptions have to be created: ramp duration for all coils, ramp delay, magnetic field strength on axis $B_{0}$, the major plasma radius $R$ and the rotational transform iota (see Fig. 4). For the entry "deenergize all coils”, however, only the high level parameter description for ramp duration has to be created.

While for each high level parameter description the unique id will be set automatically by the database, the name and properties as well as the transformation function(s) and the destination path(s) to the low level parameter(s) (goal) have to be specified (see Fig. 3). 


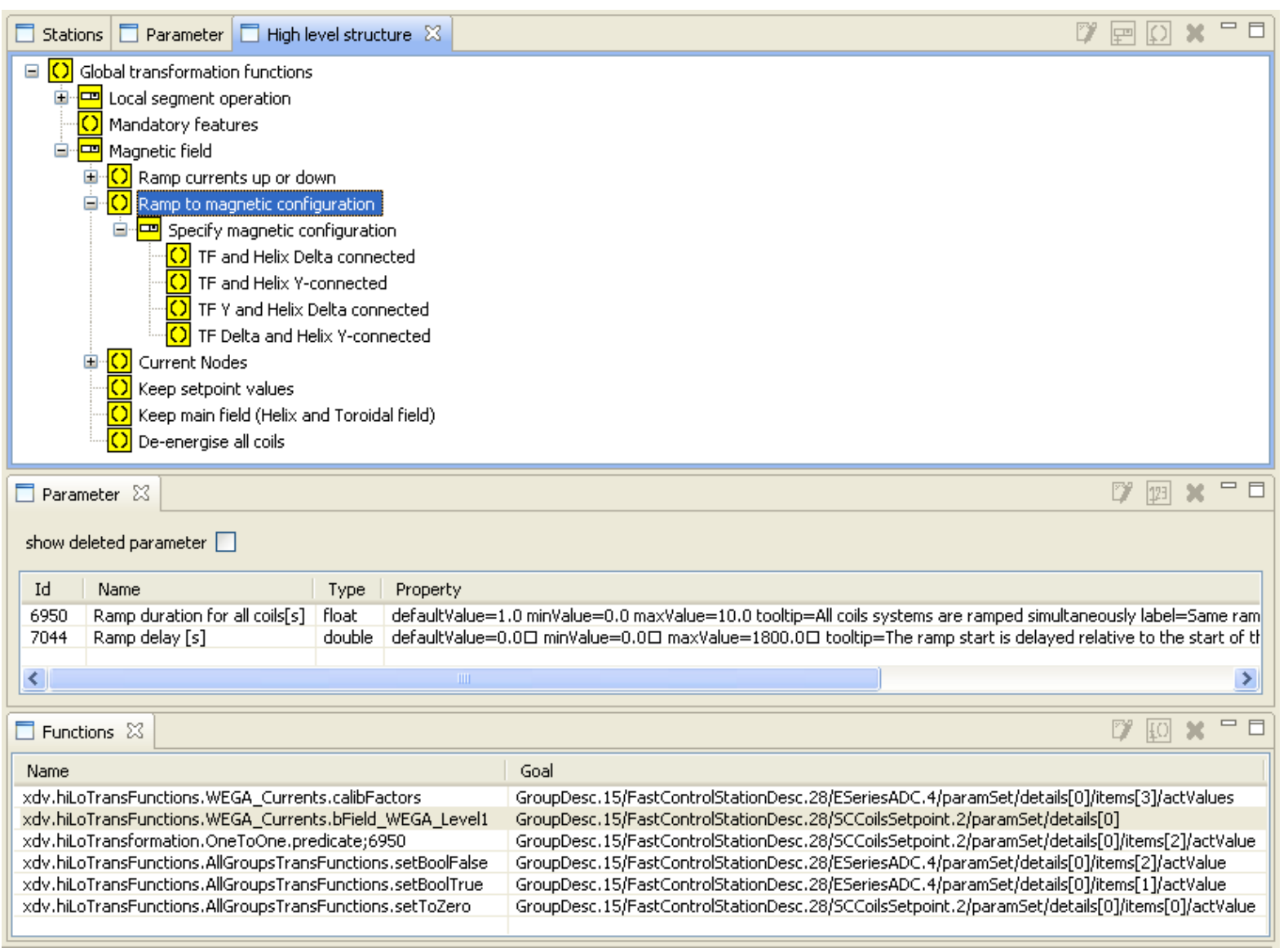

Fig. 3. Example of a high level parameter structure (top) and fully qualified specification of Java transformation function implementation (left column at bottom) and destination path to low level parameter (right column at bottom).

3) The transformation functions have to be written by the responsible physicist or technician (see Section 3.3). Adaptor stubs can be provided by a generator tool. The integration is done with the help of the CoDaC personnel.

The other high level parameters of the magnetic field component have to be setup in corresponding manner.

\subsection{Editing high level parameters of a segment description}

The segment editor is a frequently used user tool to edit the content of high level parameters of one component for single segment descriptions and to initiate the transformation and overwrite process.

This editor uses the high level parameter descriptions and the structure created with the configuration editor to build up the graphical user interface. While all parameters of a sequence are displayed, only the corresponding parameters (necessary in this context) of the selected entry are displayed in case of a choice. When another selection is made, the displayed parameters may change, see the magnetic field example in Fig. 4. The entered values are automatically checked for validity with respect to the specified range, allowed values and dependencies on other high level parameters. 


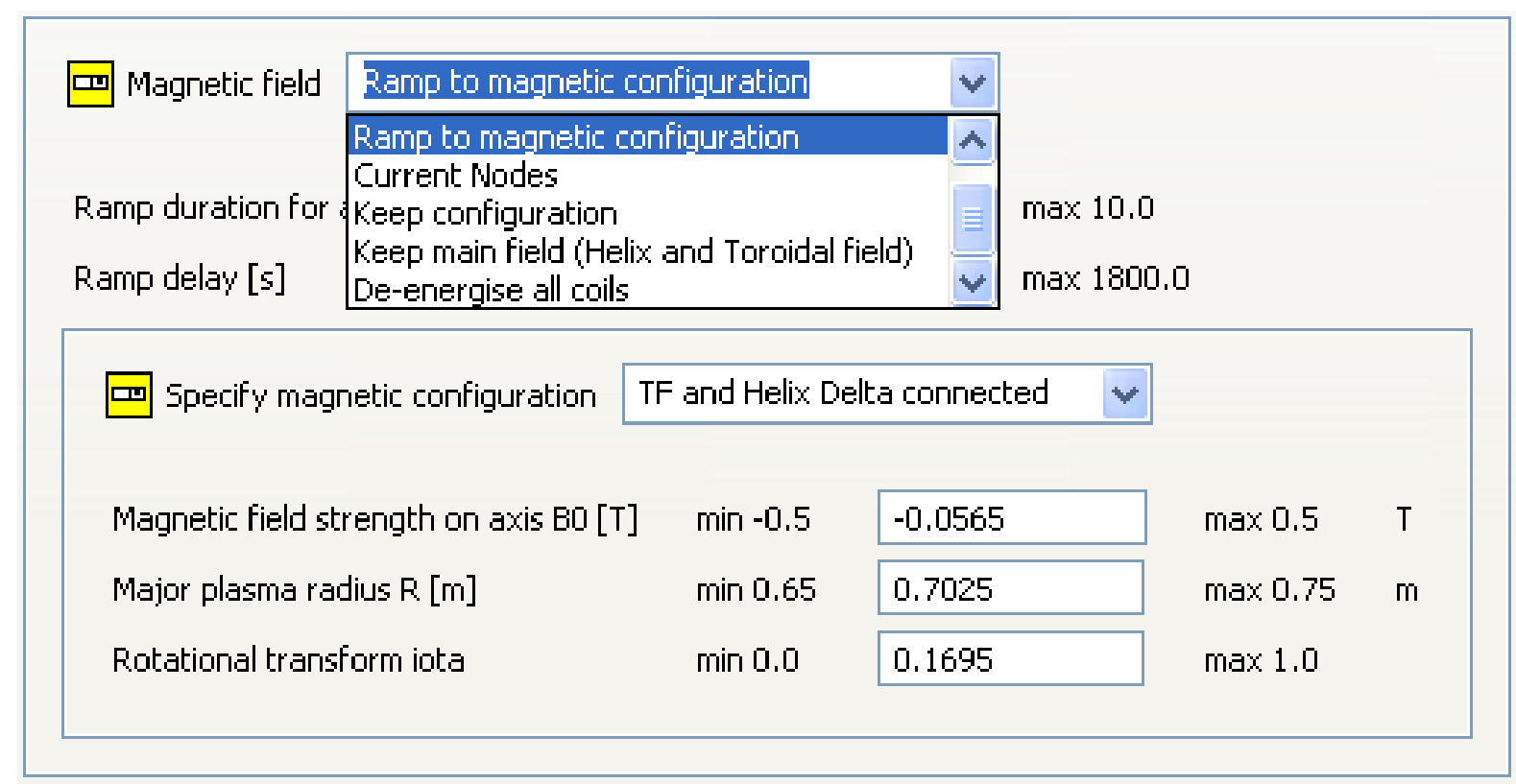

Fig. 4. Magnetic field - choice with selected entry "Ramp to magnetic configuration"; sequence with basic magnetic field parameters.

Having finished editing all high level parameters of one component for one segment, the user can initiate the transformation and overwrite process, which will then be executed automatically and create an executable segment description.

\subsection{Transformation and overwrite - from high to low level parameters}

In the transformation process, for each goal the specified transformation function will be called with all high level parameters of the selection/sequence and all high level parameters in the hierarchy below as input. The transformation function then maps the high onto low level parameters. In the overwrite process, the default values of the pattern segment description, where the goals of the transformation function point to, will be partially overwritten in the new segment description.

Besides simple one-to-one transformation functions [2], also complicated functions are already in use. For the latter ones it turned out to be best to restrict the high level input parameters to some significant quantities, which provide stable transformation results, and calculate the depending values. The full spectrum of possible high level input parameters would introduce unnecessary complexity and require much experience to avoid unstable transformations. The calculated high level parameter values will be displayed.

One example for a complicated function is the magnetic transformation function, which calculates the coil currents from the three magnetic field quantities [3]. The definition of these function and goal attributes can be seen in the second row of Fig. 3 and an extract of the implementation in Fig. 5. 


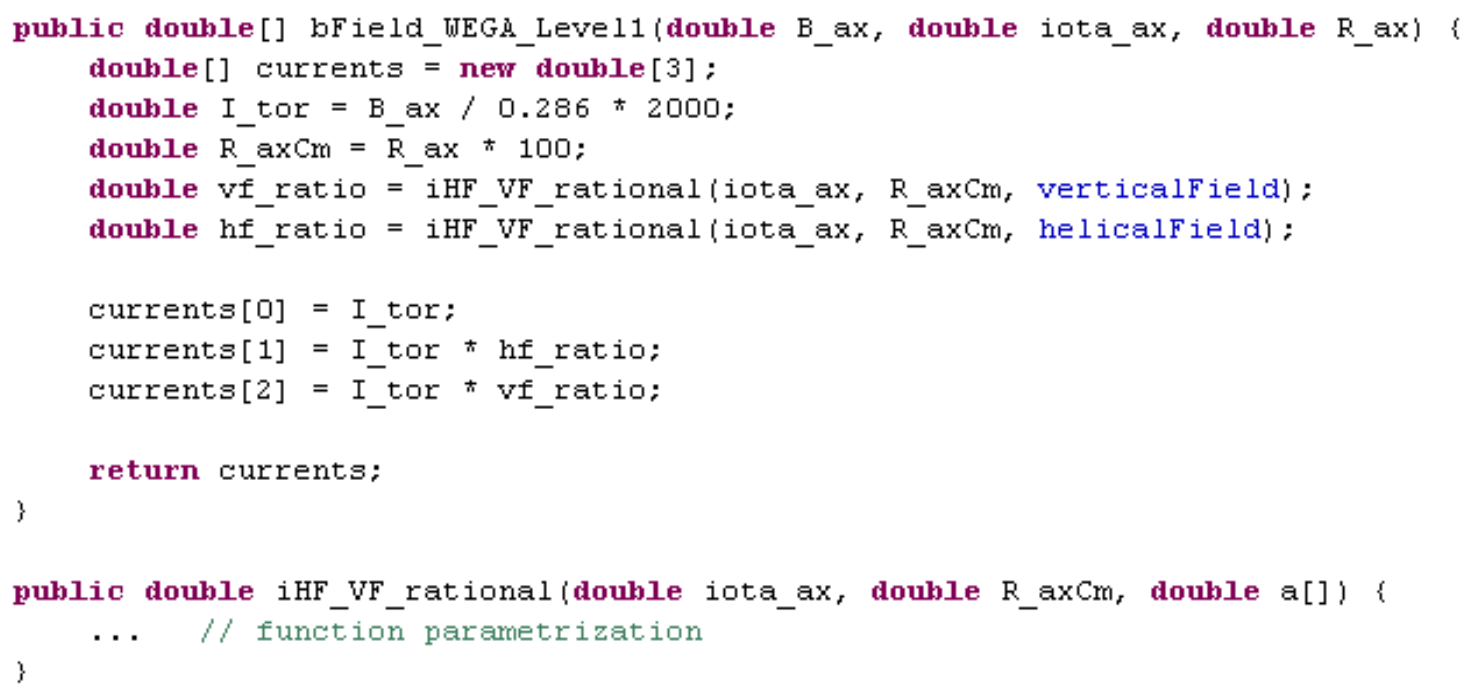

Fig. 5. Extract of user-defined transformation function (written in Java) to calculate coil currents from magnetic field quantities.

\subsection{The experiment program - putting it all together}

The WEGA magnetic field example was for high and low level parameters for one segment of one component only. For the complete experiment program, several segment descriptions for all components are needed, which have to be aggregated into a sequence. Therefore the other segment descriptions for the other components have to be created as well. Then for each segment a description containing all parameters of all components has to be created, the project segment description. When these project segment descriptions are finished, they have to be put together to a scenario description, which then again are chained up to the experiment program description.

Chaining up segment and scenario descriptions is currently done manually with the generic ConfiX editor [4]. In order to support this task in the future, a program editor remains to be developed. Currently a study is performed and a prototype is being implemented for this future editor [5]. This editor should also be used for editing conjointly used high level parameters of several segment descriptions at the same time as well as for short term editing of existing experiment program descriptions during the running experiment session.

Moreover, a validation of the planned experiment program regarding feasibility is necessary and a preview of the planned experiment run based on high level parameters is envisioned.

Besides this bottom-up editor, a top-down editor is needed for W7-X as well. This editor should, among other things, resolve dependencies of high level parameters and automatically create sequences of segment descriptions out of given physics scenarios.

\section{Outlook}

The high level parameter concept is designed to be generic, flexible and extendable so that even different levels of abstractions are possible. Currently only high level parameters for components are in use, but will follow for projects in the near future.

High level parameters cannot only be used for planning experiments, but also for other fields of application: 
- To preview of the planned experiment run, based on high level parameters.

- To start later data retrieval with high level parameters. If one e.g. wants to find all segments with a magnetic field strength on axis $B_{0}>1 \mathrm{~T}$, the parameter logs can be searched and indexed respectively for this high level parameter.

- For observation purposes like monitoring [6], e.g. the rotational transform iota values can be displayed instead of the measured coil currents.

- A nominal/actual value comparison can be done with high level parameters, e.g. for error detection.

- For diagnostic development, forward models (predictions/simulations) and inversions (analysis) are tested in the context of simulations with high level parameters. The analysis of the simulated data has to result in these high level parameters again.

Moreover, the high level parameter concept is not restricted to be used for WEGA and W7-X, but can be employed with other experiments and laboratories.

\section{References}

[1] J. Maier, T. Bluhm, P. Heimann, Ch. Hennig, H. Kroiss, G. Kühner, et al., Concurrent Object Access for the W7-X Configuration Database, this issue.

[2] H. Riemann, T. Bluhm, P. Heimann, Ch. Hennig, H. Kroiss and G. Kühner et al., 25th Symposium on Fusion Technology (SOFT-25), Rostock 2008 From a physics discharge program to device control - Linking the scientific and technical world at Wendelstein 7-X, Fusion Eng. Design 84 (2009), pp. 1598-1601 10.1016/j.fusengdes.2008.12.012.

[3] A. Sengupta, J. Geiger, M. Otte and A. Werner, Fast recovery of the magnetic vacuum configurations of the WEGA stellarator with error field effects, Plasma Phys. Control. Fusion 51 (2009) 10.1088/0741-3335/51/6/065017.

[4] H. Kühntopf, H. Kroiss, T. Bluhm, P. Heimann, Ch. Hennig and G. Kühner et al., 5th IAEA TM on Control, Data Acquisition, and Remote Participation for Fusion Research, Budapest 2005 Specialized editor for processing objects in a database to prepare discharges for WENDELSTEIN 7-X, Fusion Eng. Design 81 (2006), pp. 1741-1745.

[5] A. Spring, M. Lewerentz, T. Bluhm, P. Heimann, Ch. Hennig, G. Kühner, et al., A first W7-X experiment program editor, this issue.

[6] Ch. Hennig, P. Heimann, S. Heinzel, H. Kroiss, G. Kühner and H. Kühntopf et al., 4th IAEA TM on Control, Data Acquisition, and Remote Participation for Fusion Research, San Diego, USA, 2003 A concept of online monitoring for the Wendelstein 7-X experiment, Fusion Eng. Design 71 (2004), pp. 107-110. 